\title{
ALTERAÇÕES MORFOFUNCIONAIS INTESTINAIS NO DIABETES. REVISÃO DA LITERATURA
}

\section{INTESTINAL MORPHOFUNCTIONAL ALTERATIONS ON DIABETES. A LITERATURE REVIEW}

\author{
Jefferson Matsuiti OKAMOTO ${ }^{1}$, Cibele Boloneze LOPES ${ }^{1}$, Rafael Bulyk VEIGA ${ }^{1}$, José \\ Leonardo Rodrigues da LUZ ${ }^{1}$, Tatiane Verônica de CAMARGO ${ }^{1}$, Marcos Ricardo da \\ Silva RODRIGUES ${ }^{2}$. \\ ${ }^{1}$ Acadêmicos do Curso de Medicina. Universidade Estadual de Ponta Grossa (UEPG). Ave- \\ nida Carlos Cavalcanti, 4748, Uvaranas, 84030-900, Ponta Grossa, PR, Brasil. \\ ${ }^{2}$ Autor para contato: Professor Adjunto de Cirurgia do Aparelho Digestivo. Departamento \\ de Medicina. Universidade Estadual de Ponta (UEPG). Avenida Carlos Cavalcanti, 4748, \\ Uvaranas, 84030-900, Ponta Grossa, PR, Brasil. E-mail: okamotojeff@gmail.com
}

Data de recebimento: 01/08/2014

Data da aprovação: 10/09/2014

\section{RESUMO}

O diabetes mellitus tipo 2 (DM2) é uma doença crônica de prevalência crescente na população mundial. A fisiopatologia do DM2 é considerada multifatorial, sendo sugerido por DeFronzo (1988) que a resistência à insulina em diferentes tecidos, como o muscular e o hepático, e, de maneira mais contundente, a insuficiência das células beta pancreáticas em secretar insulina, possam ser os principais determinantes da doença. Há muitos anos, diferentes estudos mostram alterações morfológicas e funcionais do intestino delgado na evolução do diabetes, não sendo claro se a alteração pode influenciar o comportamento da doença. Este trabalho faz uma revisão da literatura relacionada ao comportamento do epitélio intestinal normal e no estado diabético.

Palavras-chave: Diabetes mellitus. Insulina. Intestino. Cirurgia bariátrica.

\begin{abstract}
Diabetes mellitus type 2 (DM2) is a chronic disease of increasing prevalence in the global population. The pathophysiology of T2DM is considered multifactorial, being suggested by DeFronzo (1988) that insulin resistance in tissues such as muscle and liver, and more striking, the failure of pancreatic beta cells to secrete insulin may be important determinants of disease. For many years, different studies show morphological and functional changes of the small intestine in the development of diabetes, being not clear whether the change can influence disease behavior. This paper reviews the literature regarding the behavior of the normal intestinal epithelium and in the diabetic state.
\end{abstract}

Keywords: Diabetes mellitus. Insulin. Intestine. Bariatric surgery. 


\section{Introdução}

Dados obtidos da Federação Internacional de Diabetes (IDF) indicam que o diabetes afeta pelo menos 285 milhões de pessoas no mundo e espera-se um aumento desse número para 438 milhões em meados de 2030. (GUARIGUATA et al., 2011). Acredita-se que a mudança dos padrões alimentares nas últimas décadas, com predominância de alimentos hipercalóricos e em grande volume, seja o responsável principal por esta pandemia. (BUCHWALD et al., 2004). Santoro et al. (2008) referem uma inadequação do trato digestivo humano ao nosso padrão alimentar, rico em alimentos com carga e índice glicêmico altos, como fator preponderante para o aumento da obesidade e diabetes na população. Outros dados fortalecem o papel do trato digestivo na evolução do diabetes, como a melhora da hiperglicemia nas primeiras semanas após cirurgias bariátricas, antes que os pacientes percam peso (SJÖSTRÖM et al., 2004), sendo sugerido a remodelação do trânsito alimentar intestinal como principal responsável pelo desfecho. Além disso, terapias que envolvem a modulação ou administração exógena de agonistas de um hormônio produzido no intestino delgado, o glucagon-likepeptide 1 (GLP-1), compreendem a última geração de medicamentos para o tratamento do distúrbio metabólico. (DRUKER, 2011). Fortalecendo ainda a importante correlação entre o trato digestivo e o desenvolvimento de DM2, Verdamet al. (2011) publicaram trabalho mostrando que pacientes obesos diabéticos têm maior massa intestinal que pacientes obesos pareados não diabéticos.

Propõe-se realizar, neste trabalho, uma breve revisão da literatura com informações pertinentes ao epitélio intestinal e o metabolismo da glicose.

\section{Fisiologia e morfologia do epitélio gastrointestinal normal}

A digestão dos açúcares, para posterior absorção, inicia durante a mastigação, sendo finalizada na borda em escova dos vilos intestinais, em sua porção apical. Esta região é rica em enzimas, como a sucrase, a maltase e a lactase, sendo que a absorção de açúcares ocorre principalmente nas porções iniciais do trato digestivo. (GANONG, 1999; LEVIN, 1994). Nos mamíferos, o transporte de glicose é realizado nos enterócitos pelas proteínas SGLT-1 e GLUT-2
(BURANTE, 1996), sendo o SGLT1 essencialmente expresso no intestino delgado. (WRIGHT et al., 2003). O SGTL1, além de transportar, funciona como um sensor de glicose, controlando a inserção membranar do GLUT-2 após ingestão alimentar. À medida que a glicose é absorvida e sua concentração no lúmen intestinal diminui, todo o sistema de sinalização é revertido, permitindo que a maioria dos GLUT-2 sejam inativados e removidos da membrana apical. (SANTER et al., 2003). Receptores GLUT-1 são normalmente abundantes no intestino fetal, porém sua concentração diminui consideravelmente na vida adulta. (THORENS, 1996).

A mucosa do epitélio gastrointestinal dos mamíferos tem a taxa de renovação mais rápida que a de qualquer tecido do corpo, e a manutenção dessa integridade depende de uma complexa interação entre processos que envolvem a proliferação, diferenciação, migração e apoptose celular. Em condições fisiológicas, células epiteliais indiferenciadas se replicam continuamente na zona de proliferação dentro de criptas e se diferenciam à medida que migram para cima da superfície luminal do colo e ápice das vilosidades no intestino delgado. (COLONY, 1983). Para manter estável o número de enterócitos, a divisão celular deve ser contrabalanceada dinamicamente pela apoptose celular na região da cripta. (BABEU et al., 2009; DUNCAN et al., 1994). O ritmo de proliferação das células intestinais sofre influência de diferentes fatores, tais como: (1) fatores genéticos, como o gene $\mathrm{Cdx} 2$, que regula tanto a taxa de diferenciação como a taxa de proliferação das células epiteliais intestinais (SUH et al.,1996); (2) fatores hormonais, como a insulina, tiroxina, hormônio do crescimento $(\mathrm{GH})$, com destaque ao êntero-hormônio GLP-2, secretado pelas células L intestinais, responsável por importante efeito trófico epitelial intestinal (VALVERDE et al., 2005); e, por último, (3) fatores luminais, como nutrientes e secreções digestivas (DAHLY et al., 2003). A presença de alimento e sua quantidade ingerida influenciam o crescimento epitelial, tanto por ação direta como pelo estímulo à secreção de êntero-hormônios. (JOHNSON, 1988). Tanto a natureza quanto a quantidade desses nutrientes interferem no processo de crescimento intestinal. Diferentes estudos mostram haver correlação direta entre a quantidade de glicose ingerida (WESER et al., 1982) ou o consumo de dietas ricas em poliaminas (LÖSER, 1999) e o 
crescimento epitelial intestinal. Ao contrário, o jejum pode diminuir o número de células mitóticas ao nível das criptas, evento que é revertido após reintrodução da dieta. (MATHERS, 1998). O crescimento epitelial promovido pelo consumo de glicose ocorre nos segmentos mais proximais do intestino, mostrando um importante papel trófico dos carboidratos, já que sua maior absorção ocorre nesse segmento. (GANONG, 1999; WESER et al., 1982).

\section{Epitélio intestinal diabético}

Há décadas, diferentes estudos relatam alterações morfológicas detectadas na presença ou no desenvolvimento do diabetes mellitus. (JERVIS et al., 1966; MILLER et al., 1977; SCHEDL et al., 1971). É observada maior quantidade de mucosa, submucosa e camada muscular externa, com destaque ao aumento da altura e volume das criptas e microvilosidades, sendo mostrado um aumento proporcional de acordo com as fases de desenvolvimento do animal. (MAYHEW et al., 1988).

No intestino diabético, a hipertrofia ou hiperplasia epitelial ocorre nos estágios iniciais da doença antes do desenvolvimento da hiperglicemia em jejum, principalmente nas porções proximais do intestino. Diferentes teorias tentam sugerir uma hipótese para o evento hiperplásico intestinal. Noda et al. (2001) realizaram estudos com ratos diabéticos, hiperfágicos em relação aos controles. Nas fases iniciais da hiperfagia, desenvolveram hiperplasia intestinal graças a uma temporária inibição da apoptose celular críptica. Sukhotniket al. (2011), analisando o papel de fatores hormonais, mostraram que a administração de insulina oral promoveu uma reversão da hiperplasia intestinal diabética, inibindo a proliferação celular e o crescimento intestinal no diabetes induzido. Histologicamente, o tratamento com insulina nos ratos mostra um decréscimo na altura das vilosidades em comparação com os animais diabéticos não tratados. Houve um efeito inibitório da insulina na proliferação do enterócito, que pode ser resultado de uma downregulation de receptores de insulina no epitélio. Apesar de ser destruída no ambiente ácido do estômago, fragmentos de insulina biologicamente ativos ou moléculas de insulina intactas podem ter modulado a proliferação celular do intestino diabético. (SUKHOTNIK et al., 2011). Os dados podem sugerir um papel dos níveis de insulina circulante assim como da expressão de receptores insulínicos na etiologia da hiperplasia intestinal diabética.

Adachiet al. (2003) realizaram estudos em diversos modelos experimentais animais de diabetes, como o Goto Kakizaki, rato não obeso e diabético. Nos experimentos foram evidenciados um aumento natural das vilosidades intestinais durante o desenvolvimento dos animais e consequente aumento da capacidade absortiva. O desfecho foi verificado tanto em ratos modelos experimentais de diabetes mellitus tipo-1 (hipoinsulinêmicos) como nos DM2, em relação ao grupo controle. Nos animais diabéticos também houve um aumento da quantidade e atividade das enzimas sucrase e isomaltase em relação aos animais controle, também justificando uma maior capacidade absortiva.

Santoro (2008) sugere a hipótese de que o nosso tubo digestivo não está preparado para as alterações que ocorreram nos nossos hábitos de vida nos últimos séculos: atividade social intensa, sedentarismo e acesso a alimentos de fácil absorção e altamente calóricos. Alimentos refinados, pobres em fibras, associados a um epitélio hiperabsortivo, deixariam o intestino distal "vazio". A consequência mais importante do intestino distal hipoestimulado seria uma menor estimulação das células L intestinais, secretoras de ênterohormônios incretínicos, encontradas principalmente nas porções distais do intestino. (VAILLANT, 1986). As incretinas são importantes para a homeostase glicêmica, já que incrementam a secreção de insulina pelo pâncreas (DRUKER, 2011). Sabe-se que diabéticos tem menores concentrações de GLP-1 plasmático, principal incretina do nosso organismo. (VILSBOLL et al., 2001). A baixa concentração do êntero-hormônio, dentre outras causas, pode ser resultado de uma maior capacidade absortiva do intestino proximal diabético. Em humanos, Verdum et al. (2011), através da quantificação da citrulina sérica, aminoácido cuja concentração pode predizer o volume de massa intestinal funcionante, mostraram que pacientes obesos diabéticos possuem de forma significante maior massa intestinal que obesos não diabéticos. Dyeret al. (2002) justificam a maior capacidade absortiva intestinal pela maior expressão de transportadores de monossacarídeos SGLT1, GLUT5, GLUT2 na mucosa intestinal diabética. Ainda estudando humanos, através da utilização de próteses plásticas (EndoBarrier) de aplicação endoscópica, 
que impedem ou diminuem o contato do alimento com a mucosa intestinal proximal, foram observadas melhora significativa nos níveis glicêmicos em jejum, resistência à insulina e glicemia pós-prandial. (DE MOURA, 2011; ESCALONA, 2012). Sugerese neste trabalho que a prótese pode, dentre outras formas, melhorar o metabolismo da glicose através do auxílio ao alimento para ultrapassar a "barreira absortiva" do intestino proximal hipertrofiado.

\section{Considerações finais}

O papel do trato gastrointestinal no desenvolvimento do diabetes está cada vez mais estabelecido. Novos estudos devem ser realizados no sentido de determinar se o controle da hiperplasia intestinal diabética e, consequentemente, de sua capacidade absortiva aumentada pode alterar o comportamento evolutivo da doença.

\section{Referências}

ADACHI, T.; MORI, C.; SAKURAI, K.; SHIHARA, N.; TSUDA, K.; YASUDA,K. Morphological changes and increased sucrose and isomaltase activity in small intestines of insulin- deficient and type 2 diabetic rats. Endocrinology Journal, v. 50, n.3, p.271-9, 2003.

BABEU, JP.; DARSIGNY, M.; LUSSIER, CR.; BOUDREAU, F. Hepatocyte nuclear factor 4alpha contributes to an intestinal epithelial phenotype in vitro and plays a partial role in mouse intestinal epithelium differentiation. American Journal of Physiology Gastrointestinal and Liver Physiology, v. 297, p.124-134, 2009.

BUCHWALD, H.; ESTOK, R.; FAHRBACH, K. et al. Weight and type 2 diabetes after bariatric surgery: systematic review and meta-analyses. The Journal of Medicine, v.122, n.3, p. 248-56, 2004.

BURANTE, C. Facilitative glucose transport. In: BITTAR, E. Principles of Medical Biology. Amsterdam: Elsevier BV, 1996, p.67-86.

COLONY, PC. Successive phases of human fetal intestinal development. In: KRETCHMER, N, MIINKOWSKI, A. Nutritional adaptation of the gastrointestinal tract of the newborn. New York: Vevey/Raven Press, 1983, p.3-28.

DAHLY, EM.; GILLINGHAM, MB.; GUO, Z.; MURALI, SG.; NELSON, DW.; HOLST, JJ.; NEY, DM. Role of luminal nutrients and endogenous GLP-2 in intestinal adaptation to mid-small bowel resection. American Journal of Physiology - Gastrointestinal and Liver Physiology, v.284, n.4, p.670-82, 2003.

DEFRONZO, RA. Lilly Lecture: The triunvirate: Beta cell, muscle, liver: a collusion responsible for NIDDM. Diabetes, p. 667-87, 1988.

DE MOURA, EG., ORSO, IR.; MARTINS, BC.; LOPES, GS.; DE OLIVEIRA, SL.; GALVAO-NETO, MP.; MANCINI, MC.; SANTO, MA.; SAKAI, P.; RAMOS, AC. et al. Improvement of insulin resistance and reduction of cardiovascular risk among obese patients with type 2 diabetes with the duodenojejunal bypass liner. ObesSurg, v.21, p.941-47, 2011.

DRUKER, DJ. Incretin-based therapy and the quest for sustained improvements in beta cell health. Diabetes care, v.34, p.2133-35, 2011.

DUNCAN, AS.; MANOVA, K.; CHEN, WS.; HOODLESS, P.; WEINSTEIN, DC.; BACHVAROVA, RF.; DARNELL, JE Jr. Expression of transcription factor HNF-4 in the extraembryonic endoderm, gut, and nephrogenic tissue of the developing mouse embryo: HNF-4 is a marker for primary endoderm in the implanting blastocyst. ProcNatlAcadSciUSA ,v. 91, p. 7598-7602, 1994.

DYER, J.; WOOD, IS.; PALEJWALA, A.; ELLIS, A.; SHIRAZI BEECHEY, SP. Expression of monosaccharide transporters in intestine of diabetic humans. American Journal of Physiology - Gastrointestinal and Liver Physiology, v.282, p.241-48, 2002.

ESCALONA, A.; PIMENTEL, F.; SHARP, A.; BECERRA, P.; SLAKO, M.; TURIEL, D.; MUNOZ, R.; BAMBS, C.; GUZMAN, S.; IBANEZ, L et al. Weight loss and metabolic improvement in morbidly obese subjects implanted for 1 year with an endoscopic duodenal-jejunal bypass liner. Ann Surg, v.255, p.1080-85, 2012.

GANONG, WF. Review of Medical Physiology.19th edn. East Norwalk, CT, USA; Appleton \& Lange, 1999.

GUARIGUATA, L.; WHITING, D.; WEIL, C.; UNWIN, N. The International Diabetes Federation. Diabetes atlas methodology for estimating global and national prevalence of diabetes in adults. Diabetes Res ClinPract, v.94, n.3, p.322-32, 2011.

JERVIS, EL.; LEVIN, RJ. Anatomic adaptation of the alimentary tract of the rat to the hyperphagia of chronic alloxan-diabetes. Nature, v.210, p.391-93, 1966.

JOHNSON, LR. Regulation of gastrointestinal mucosal growth. Physiol Rev, v.68, p.456-502, 1988.

LEVIN, RJ. Digestion and absorption of carbohydratesfrom molecules and membranes to humans. Am J ClinNutr, v.59, p.690-8, 1994.

LÖSER, C.; EISEL, A.; HARMS, D.; FÖLSCH, UR. Dietary polyamines are essencial luminal growth factors for small intestinal and colonic mucosal growth and development. Gut, v.44, p.12-16, 1999.

MATHERS, JC. Nutrient regulation of intestinal 
proliferation and apoptosis. Proceedings of the Nutrition Society, v.57, p.219-23, 1998.

MAYHEW, T.M.; CARSON, F.L. Mecanism of adaptation in rat small intestine: regional difference in quantitative morphology during normal growth and experimental hypertrophy. Anatomy Journal, v.164, n.1, p. 189-200, 1988.

MILLER, DL. ; HANSON, W.; SCHEDL, HP.; OSBORNE, JW. Proliferation rate and transit time of mucosal cells in small intestine of the diabetic rat. Gastroenterology, v.73, p.1326-1332, 1977.

NODA, T.; IWAKIRI, R.; FUJIMOTO, K.; YOSHIDA, T.; UTSUMI, H.; SAKATA, H.; HISATOMI, A.; AW, TY. Suppression of apoptosis is responsible for increased thickness of intestinal mucosa in streptozotocin - induced diabetic rats. Metabolism, v.50, n.3, p.259-64, 2001.

SANTER, R.; HILLEBRAND, G.; STEINMANN, B.; SCHAUB, J. Intestinal glucose transport: Evidence for a membrane traffic-based pathway in humans. Gastroenterol, v.124, p.34-9, 2003.

SANTORO, S. Is the Metabolic syndrome a disease of the foregut? Yes, excessive foregut. Ann Surg, v.247, p.10741075, 2008.

SCHEDL, H.; WILSON, H. Effects of diabetes on intestinal growth in rat. Journal of experimental zoology, v.176, p.487-496, 1971.

SJÖSTRÖM, L.; LINDROOS, A.K.; PELTONEN, M et al. Lifestyle, diabetes, and cardiovascular risk factors 10 years after bariatric surgery. N. Engl. J. Med, v.351, p.2683, 2004.

SUH, E.; TRABER, P.G. An intestine-specific homeobox gene regulates proliferation and differentiation. Mol CellBiol, v.16, n.2, p.619-25, 1996.

SUKHOTNIK, I.; SHAMIR, R.; BASHENKO, Y.; MOGILNER, JG., CHEMODANOV, E.; SHAOUL, R.; CORAN, AG.; SHEHADEH, N. Effect of oral insulin on diabetes-induced intestinal mucosal growth in rats. DigDisSci, v.56, p.2566-74, 2011.

THORENS, B. Glucose transporters in the regulation of intestinal, renal, and liver glucose fluxes. American Journal of Physiology - Gastrointestinal and Liver Physiology. v. 270, n.4, p.541-53, 1996.

VAILLANT, CR.; LUND, PK. Distribution of glucagon-like peptide $I$ in canine and feline pancreas and gastrointestinal tract. J Histochem Cytochem, v.34, p.1117-21, 1986.

VALVERDE， I.;PUENTE， J.; MARTIN-DUCE， A. Changes in glucagon-like peptide-1 (GLP-1) secretion after biliopancreatic diversion or vertical banded gastroplasty in obese subjects. ObesSurg, v.15, n.3, p. 387-97, 2005.
VERDAN, FJ.; GREVE, JW.; ROOSTA, S.; VAN EIJK, H.; BOUVY, N.; BUURMAN, WA; RENSEN, SS. Small intestinal alterations in severely obese hyperglycemic subjects. J ClinEndocrinolMetab, v.96, n.2, p. 379-83, 2010 .

VISBLSBØLL, T.; KRARUP, T.; DEACON, CF.; MADSBAD, S.; HOLST, JJ. Reduced postprandial concentrations of intact biologically active glucagon-like peptide 1 in type 2 diabetic patients. Diabetes, v.50, p.60913,2001 .

WESER, E.; BABBITT, J.; HOBAN, M.; VANDEVENTER, A. Intestinal adaptation: different growth responses to disaccharides compared with monosaccharides in rat small bowel. Gastroenterology, v.91, n.6, p.1521-7, 1986.

WRIGHT, E.M.; MARTIN, M.G.; TURK, E. Intestinal absorption in health and disease - sugar. Best Pract Res Clin Gastroenterol. v.17, n.6, p.943-56, 2003. 